\title{
Social dynamics of financial networks
}

\author{
Teruyoshi Kobayashi ${ }^{1}$ and Taro Takaguchi ${ }^{2,3,4^{*}}$
}

\author{
${ }^{*}$ Correspondence: \\ taro.takaguchi.cp@gmail.com \\ ${ }^{2}$ National Institute of Information \\ and Communications Technology, \\ Tokyo, Japan \\ ${ }^{3}$ National Institute of Informatics, \\ Tokyo, Japan \\ Full list of author information is \\ available at the end of the article
}

\begin{abstract}
Recurrent interactions between agents play an essential role in the organization of a dynamic complex system. While intensive researches have been done on social systems formed by human interactions, dynamical rules are not well understood in economic systems. Here we study the evolution of financial networks and show that repeated interactions between financial institutions taking place at the daily scale are characterized by social communication patterns of humans emerging at higher time scales. The "social" dynamics of financial interactions are highly stable and little affected by external shocks such as the occurrence of the global financial crisis. A dynamic network model based on random pairwise matching accurately explains the observed daily dynamical patterns. The observed similarity between social and financial interactions gives us previously unknown stylized facts about a financial system, which could lead to a deeper understanding of the fundamental source of systemic risk.
\end{abstract}

Keywords: Financial networks; Systemic risk; Temporal networks; Scaling; Fitness model

\section{Springer}

\section{Introduction}

Financial systemic risk is one of the most serious threats to the global economy. The global financial crisis of 2007-2009 showed that a failure of one bank can lead to a financial contagion through a complex web of financial linkages, which are created by everyday transactions among financial institutions [1-6]. Even after the crisis, many countries have experienced a prolonged recession, the so-called Great Recession, showing that the social cost of a financial crisis can be enormous [7, 8]. Evaluating and controlling systemic risk has therefore been recognized as one of the greatest challenges for interdisciplinary researchers across different fields of science [9-12].

In the modern financial system, interbank markets play a fundamental role, in which banks lend to and borrow from each other (hereafter, we refer to all types of financial institutions as "banks" for brevity). Lending and borrowing in interbank markets are necessary daily tasks for banks to smoothen their liquidity management [13], but at the same time, they also form the center of a global web of interconnected risk; shocks to interbank markets may spill over to other parts of the global financial system through the financial linkages to which banks are connected [10, 14, 15]. Many previous studies attempt to assess systemic risk by simulating different scenarios of cascading bank failures on both real [16-20] and synthetic interbank credit networks [15, 21-26]. Studies of financial cascades based on synthetic networks often assume a particular static structure,

(c) The Author(s) 2018. This article is distributed under the terms of the Creative Commons Attribution 4.0 International License (http://creativecommons.org/licenses/by/4.0/), which permits unrestricted use, distribution, and reproduction in any medium, provided you give appropriate credit to the original author(s) and the source, provide a link to the Creative Commons license, and indicate if changes were made. 
such as random [22, 23], bipartite [15, 27], and multiplex structures [25, 26], successfully revealing that the structural property affects the likelihood of default cascades to a large extent. However, since the great majority of real-world interbank transactions are in fact overnight $[13,28]$, interbank networks should be treated as dynamical systems with their structure changing on a daily basis. This temporal nature of real interbank networks inevitably limits the practical usefulness of the conventional static approach to systemic risk. Nevertheless, we still have little knowledge about how the structural characteristics of daily networks evolve over time. It has long been believed that the dynamics of interbank networks is random and thus has no meaningful regularity at the daily scale [29]. How the current network structure is correlated with the past structures, or more specifically, how banks choose current trading partners based on their trading history, is still unknown. The ambiguity of the structural dynamics of interbank networks itself may also become a source of systemic risk by veiling the complexity of interconnectivity [30]. The current lack of studies on the mechanics of real interbank networks is in stark contrast to the abundance of research on their static property [16, 19, 31-34].

The main aim of this work is to uncover fundamental dynamics governing real interbank networks at both local and system-wide scales. For this purpose, we first seek dynamical regularities that would characterize interaction patterns between individual banks by examining millions of overnight transactions conducted in the Italian interbank market during 2000-2015 [35]. We discover that there exist explicit interaction patterns that rule daily bank-to-bank transactions, which turn out to be essentially the same as the patterns characterizing human social communication; that is, banks trade with each other in the same way that people interact with friends through phone calls and face-to-face conversations [36, 37]. In fact, those "social" interaction patterns of banks have been surprisingly stable over time, even amid the global financial crisis. On top of local interactions between banks, there emerges a system-wide scaling relationship between the numbers of banks and transactions, just as the number of phone-call pairs scales superlinearly with the size of population [38].

To explain the origin of social dynamics in interbank networks, we develop a model that generates a sequence of synthetic daily networks from which the observed dynamical patterns simultaneously emerge at both local and network scales. Our discovery of the fundamental mechanism underpinning the daily evolution of interbank networks will enhance the predictability of systemic risk and provide an important step toward the realtime management of financial stability.

\section{Results}

The dataset to be analyzed in this work is the time series of daily networks identified from the time-stamped data of interbank transactions conducted in the Italian interbank market during 2000-2015 (see Methods for data description). The daily interbank networks have directed edges originating from lending banks to borrowing banks. One may regard the amount of funds transferred from a lender to a borrower as edge weights, but here we regard the daily networks as unweighted, since we found that the dynamics of edge weights can be understood independently from the edge dynamics themselves (see Methods for the analysis of edge weights).

An important general observation regarding the dynamics of daily networks is that both the network size $N$ and number of edges $M$ have followed downward trends (Fig. 1(a) 

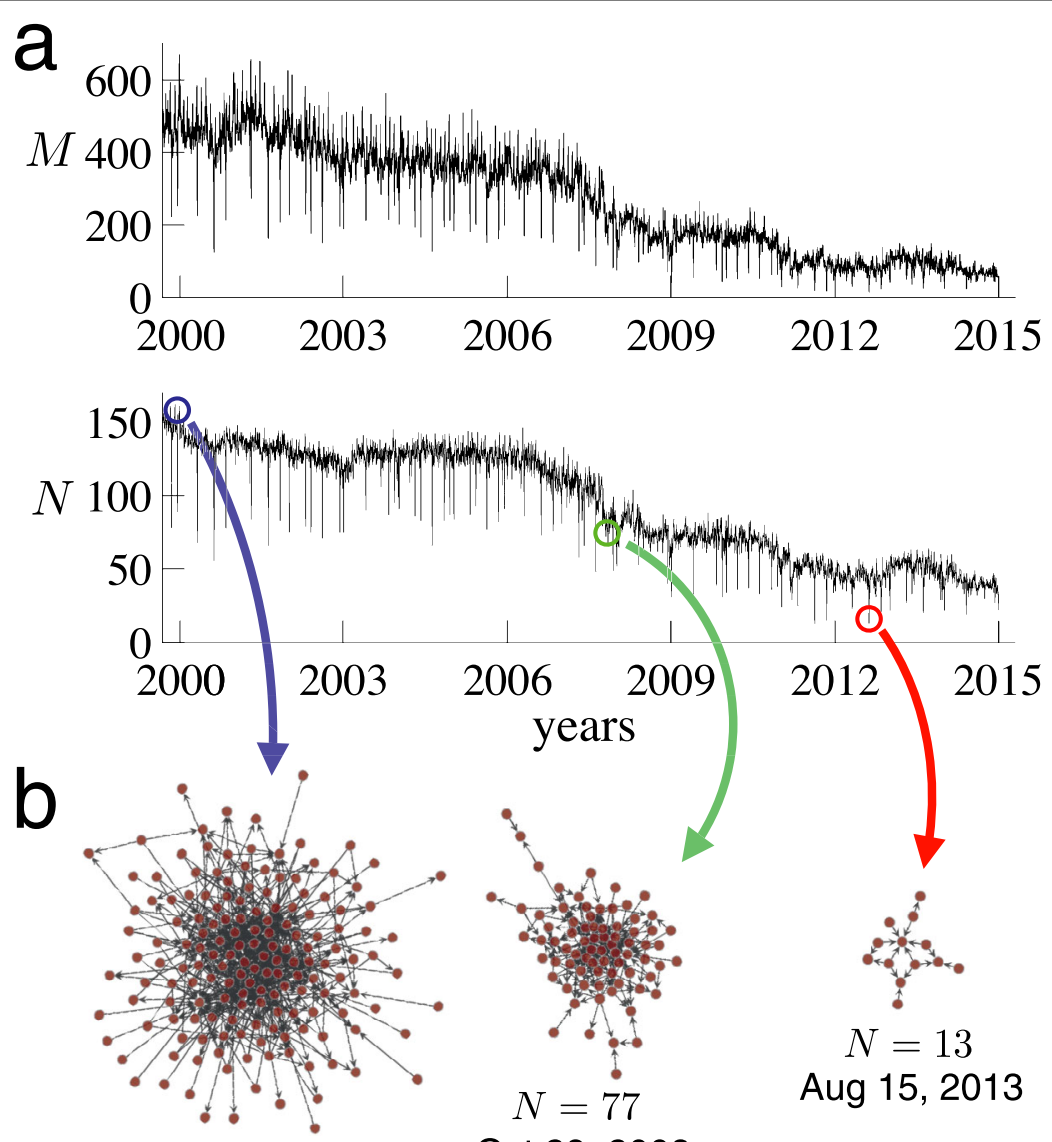

$N=162$

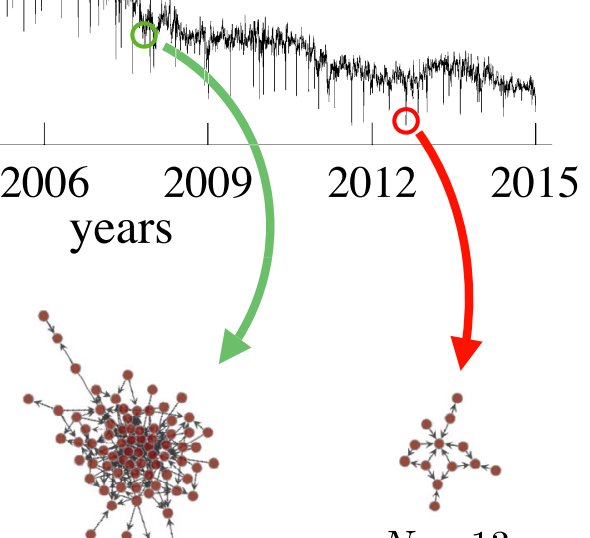

$$
N=77
$$

$N=13$

Oct 29, 2008

Nov 23, 2000

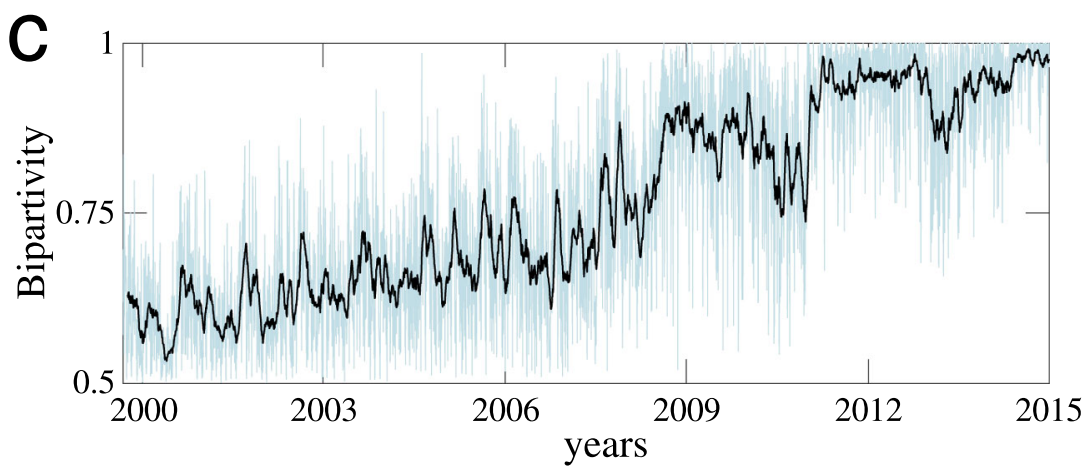

Figure 1 Time series of daily networks. (a) Daily time series of the number of edges $M$ (upper) and the number of banks $N$ (lower). Most of the downward spikes in $N$ and $M$ are due to the national holidays in Italy. (b) Visualization of the largest (left), a middle-sized (middle) and the smallest (right) daily networks (visualized by graph-tool [65]) (c) Time series of bipartivity. Bipartivity is a measure of bipartite structure defined as $\sum_{i=1}^{N} \cosh \left(\hat{\lambda}_{i}\right) / \sum_{i=1}^{N} \exp \left(\hat{\lambda}_{i}\right)$, where $\left\{\hat{\lambda}_{i}\right\}$ denotes the eigenvalues of the adjacency matrix. The bipartivity measure takes 1 if the network is fully bipartite and 0.5 if a complete graph [66]. Since the bipartivity is defined for undirected graphs, we ignore the directionality of edges. Black line represents the moving average with 20-day smoothing window

and (b)). This led the networks closer to a bipartite structure between pure lenders and pure borrowers [39] (Fig. 1(c) and Table 1), entailing a non-negligible turnover in the set of banks participating in each daily network (see the turnover rate in Table 2 and Fig. S1(a) 
Table 1 Fraction of each bank type in the data. "Pure lender" ("pure borrower") denotes the banks that lend to (borrow from) but never borrow from (lend to) other banks

\begin{tabular}{lllll}
\hline & All & $2000-2006$ & $2007-2009$ & $2010-2015$ \\
\hline Pure lender & 0.556 & 0.553 & 0.571 & 0.558 \\
Pure borrower & 0.335 & 0.300 & 0.318 & 0.380 \\
Others & 0.110 & 0.147 & 0.111 & 0.062 \\
\hline
\end{tabular}

Table 2 Summary statistics of the daily interbank networks. $\bar{N}$ and $\bar{M}$ denote the average numbers of active banks and edges in the daily networks, respectively. The turnover rate is the average of the Jaccard distance $1-\left|I_{t} \cap I_{t-1}\right| /\left|I_{t} \cup I_{t-1}\right|$, where $I_{t}$ is the set of active banks on day $t$. See caption of Fig. 1(c) for a description of bipartivity

\begin{tabular}{lllll}
\hline & All & $2000-2006$ & $2007-2009$ & $2010-2015$ \\
\hline$\#$ days & 3922 & 1618 & 767 & 1537 \\
$\bar{N}$ & 94.23 & 129.69 & 98.92 & 54.56 \\
$\bar{M}$ & 266.40 & 409.75 & 268.40 & 114.51 \\
Turnover rate & 0.22 & 0.18 & 0.22 & 0.26 \\
Bipartivity & 0.77 & 0.64 & 0.78 & 0.92 \\
\hline
\end{tabular}

and (b) in supplementary materials (SM)). Table 2 summarizes the basic statistics, in which we divide the entire sample period into three subsample periods to observe whether a structural change around the global financial crisis in 2007-2009 is present.

\subsection{Dynamical patterns of daily networks}

The downward trends in $N$ and $M$, along with the intermittent spikes, left a broad range of daily combinations $(N, M)$, which allows us to reveal a superlinearity, $M \propto N^{1.5}$ (Fig. 2(a)). This suggests that the average degree of a daily network increases with order $\sqrt{N}$, since $\langle k\rangle=M / N \propto \sqrt{N}$. It should be noted that the fact that $M$ is given as a power-law function of $N$ is similar to a widely observed phenomenon in social networks, called superlinear scaling, in which the number of edges scales superlinearly with the number of nodes across different locations [38, 40-42].

In addition to the macroscopic dynamics of $N$ and $M$, we also find microdynamics of individual edges: edge duration and interval time. We define duration $\tau$ as the number of successive business days on each of which a lender and a borrower performs at least one transaction (i.e., directionality is taken into account). Aggregated over all trading pairs, $\tau$ follows a power-law distribution whose complementary cumulative distribution function (CCDF, see Table 3 for the list of abrreviations hereafter) has an exponent between 2.5 and 2.9 (Fig. 2(b) and Fig. S2 in SM. The exponents are estimated using the method proposed in Refs. $[43,44])$. Similar power-law distributions are observed when we redefine $\tau$ as the duration of individual banks' successive trading activity either for lending, borrowing, or both (Fig. S3 in SM).

On the other hand, the interval time $\Delta \tau$ for a bank pair is defined as the interval length between two consecutive transactions during which the bank pair performs no transactions. In contrast to $\tau, \Delta \tau$ does not follow a power-law distribution while it still shows a long-tailed behavior (Fig. 2(c)). The interval distribution fits well with a Weibull distribution up to a certain cut-off level (Fig. 2(c), Inset. See Methods for details on the fitting method $[45,46])$.

We observe that the distributions of $\tau$ and $\Delta \tau$ have been quite stable throughout the whole data period. This observation is notable, not only because $N$ and $M$ continually 

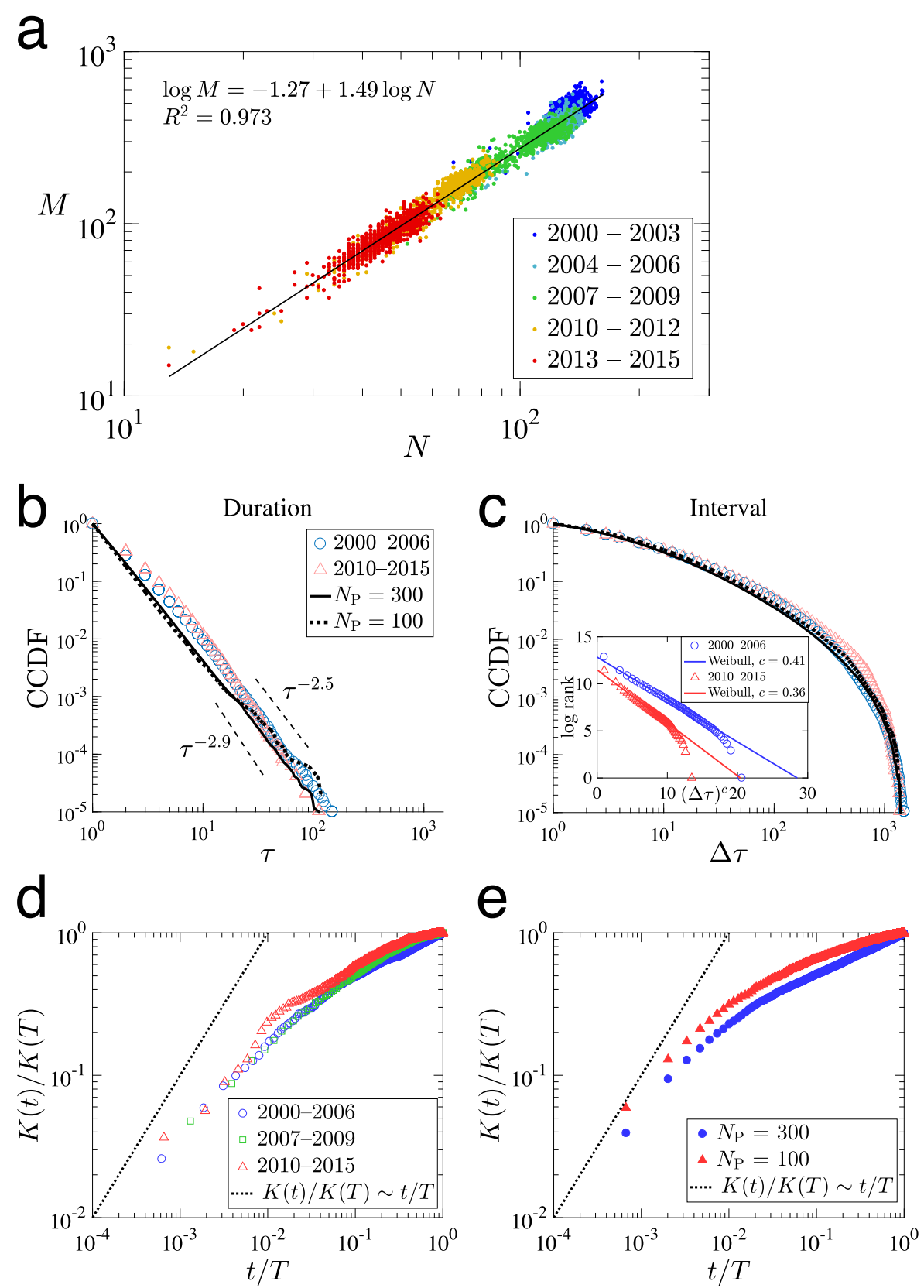

Figure 2 Dynamical patterns of daily networks. (a) $M$ is a power-law function of $N$. A dot corresponds to a daily network, and solid line represents a log-linear regression estimated by the ordinary least squares. (b) Distribution of consecutive trading days, $\tau$, for a bank pair, aggregated over all pairs. Blue circles and red triangles indicate the empirical distributions for 2000-2006 and 2010-2015, respectively. Solid and dotted lines represent simulated distributions with $N_{\mathrm{P}}=300$ and 100, respectively. (c) Distribution of interval time, $\Delta \tau$, between two consecutive transactions for a bank pair, aggregated over all pairs. Inset: log-rank plot of $(\Delta \tau)^{c}$ indicated by a Weibull distribution with estimated parameter $c$ (symbols), and its theoretical values (lines). (d) Time series of the empirical aggregate degree $K(t)$ (averaged over all banks) normalized by its terminal value $K(T)$ and (e) the simulated aggregate degree

fluctuate at the daily resolution over the course of the decreasing trends (Fig. 1(a)), but also because a large fraction of the set of participating banks changes day to day (Fig. S1(a) and (b)). The high metabolism of the interbank market suggests that the stationarity of $\tau$ and 
Table 3 List of abbreviations

\begin{tabular}{ll}
\hline $\mathrm{ON}$ & overnight \\
$\mathrm{ONL}$ & overnight large \\
$\mathrm{CCDF}$ & complementary cumulative distribution function \\
OLS & ordinary least squares \\
\hline
\end{tabular}

$\Delta \tau$ is not necessarily attributed to the presence of steady relationships between particular banks.

The fact that banks' behavior is characterized by heavy-tailed distributions of duration and interval time might imply that banks are social. Another property that reinforces the social aspect of banks is found in the growth rate of aggregate degree $K(t)$, denoted by the average cumulative number of unique trading partners up to time $t[37,47]$. The normalized aggregate degree, defined by $K(t) / K(T)$, grows sublinearly in time (Fig. 2(d)), meaning that the rate at which banks find a new partner tends to decrease over time. Note that such sublinear growth patterns have been reported for the mobility pattern of mobilephone users [47] and for contact networks of human individuals formed via face-to-face interactions [37].

\subsection{Model of daily network evolution}

The above findings show that the dynamical patterns of interbank transactions are robust across different periods, which leads us to consider that a universal mechanism generating daily interbank networks might exist. Here, we show that the emergence of these regularities can be reconstructed by a dynamical generalization of the fitness model [ 48 , 49].

First, we show that variations in the system size of a simple fitness model can explain the empirical superlinear relation $M \propto N^{1.5}$. For ease of exposition, suppose for the moment that the networks are undirected. In the fitness model, fitness value $a_{i} \in[0,1]$ is assigned to bank $i\left(1 \leq i \leq N_{\mathrm{P}}\right)$, where $N_{\mathrm{P}}$ represents the potential market size, given by the number of banks that may perform transactions during a day. In the context of interbank transactions, fitness value $a_{i}$ can be interpreted as the activity level, or willingness, of bank $i$ to trade. The probability that an edge is formed between $i$ and $j$ is given by $p_{i j}=\left(a_{i} a_{j}\right)^{\alpha}(\alpha \geq 1)$. For each network generated by this rule, $N$ and $M$ denote the number of active banks with at least one edge (thus $N \leq N_{\mathrm{P}}$ ) and the total number of edges, respectively.

By generating model networks with $N_{\mathrm{P}}$ varying from 20 to 300 for a given $\alpha \in[2,8]$, there arises a scaling relation $M \propto N^{\beta}$ with $1<\beta<2$ (symbols in Fig. 3). In previous studies [4850], a theoretical analysis of the fitness model predicted $M \propto N^{2}$, which differs from both our empirical observations (Fig. 2(a)) and numerical simulations (Fig. 3). In fact, this discrepancy is explained by the presence of isolated banks. In this model, the probability of a bank being isolated (i.e., no edges attached), defined by $q_{0}$, is given by a function of $N_{\mathrm{P}}$ :

$$
q_{0}\left(N_{\mathrm{P}}\right)=\frac{1}{\alpha}(\alpha+1)^{\frac{1}{\alpha}}\left[\mathrm{B}\left(N_{\mathrm{P}}, \frac{1}{\alpha}\right)-\mathrm{B}_{1-\frac{1}{\alpha+1}}\left(N_{\mathrm{P}}, \frac{1}{\alpha}\right)\right]
$$

where $\mathrm{B}(x, y)$ and $\mathrm{B}_{z}(x, y)$ are beta and incomplete beta functions, respectively (see Methods for derivation). Consequently, $N$ and $M$ are given by

$$
\left\{\begin{array}{l}
N=\left(1-q_{0}\left(N_{\mathrm{P}}\right)\right) N_{\mathrm{P}}, \\
M=\left\langle\left(a_{i} a_{j}\right)^{\alpha}\right\rangle \frac{N_{\mathrm{P}}\left(N_{\mathrm{P}}-1\right)}{2} .
\end{array}\right.
$$




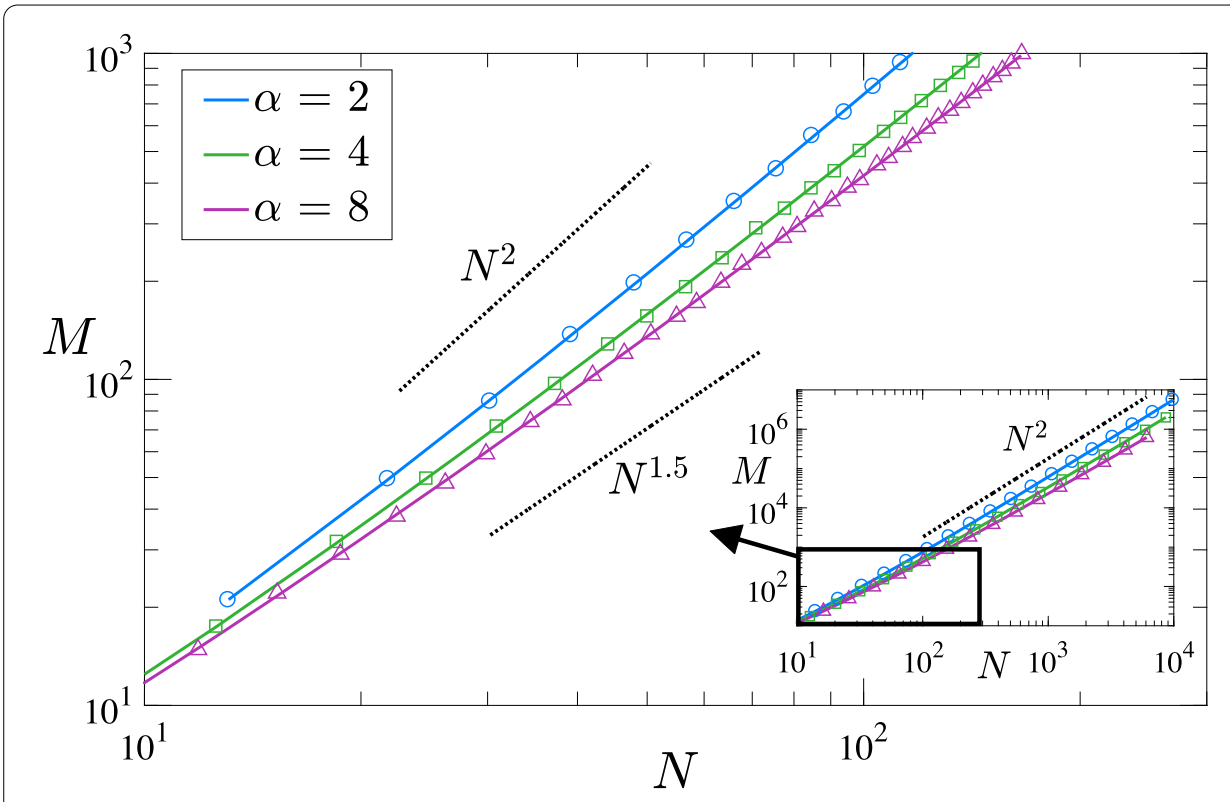

Figure 3 Superlinear relation in the fitness model. Solid lines denote the theoretical values indicated by Eq. (2), while symbols represent the corresponding simulation results (average of 500 runs). Scaling relation $M \propto N^{\beta}$ with $1<\beta<2$ emerges by varying $N_{\mathrm{P}}$ when $N_{\mathrm{P}}$ is small (approximately $20 \leq N_{\mathrm{P}} \leq 300$ ). Inset: increasing $N_{\mathrm{P}}$ to 10,000 restores quadratic scaling $M \propto N^{2}$ since $q_{0} \approx 0$ and thereby the finite-size effect disappears

Since $q_{0}\left(N_{\mathrm{P}}\right) \rightarrow 0$ as $N_{\mathrm{P}} \rightarrow \infty, N \simeq N_{\mathrm{P}}$ and $M \simeq\left\langle\left(a_{i} a_{j}\right)^{\alpha}\right\rangle N(N-1) / 2 \propto N^{2}$ hold true for a sufficiently large $N_{\mathrm{P}}$, which recovers the quadratic scaling shown in the previous studies [48-50]. However, for the range of network sizes observed from the data, $q_{0}\left(N_{\mathrm{P}}\right)$ is not negligible. A combination of $(N, M)$ derived from (2) for given values of $N_{\mathrm{P}}$ perfectly fits the simulation result (lines in Fig. 3).

While the empirical superlinear relation $M \propto N^{1.5}$ can be explained by variations in $N_{\mathrm{P}}$, this simple model is unable to reproduce the distributions of $\tau$ and $\Delta \tau$ (Fig. 2(b) and (c)) and the sublinear growth of $K(t)$ (Fig. 2(d)), because these characteristics come from the effect of memory in the formation of links between banks [28]. To capture the memory effect, we now introduce a fluctuation in fitness $a_{i}$. The dynamic network formation in our model proceeds by repeating the following two steps: (i) edge creation between banks and (ii) update of each bank's activity level $a_{i, t}$. We consider three bank types: pure lenders, pure borrowers, and bidirectional traders. Pure lenders (pure borrowers) are the banks that may lend to (borrow from) but never borrow from (lend to) other banks. To take into account the fact that the interbank structure is almost perfectly bipartite when the network size is small (Fig. 1(c)), we assume that bidirectional traders may lend only to pure borrowers and borrow only from pure lenders. The fraction of each bank type is given as $\left(f_{\mathrm{L}}, f_{\mathrm{B}}, f_{\mathrm{D}}\right)=(0.56,0.34,0.1)$ for pure lenders, pure borrowers, and bidirectional traders, respectively, based on the empirical average (Table 1). The type assigned to each bank is fixed throughout the simulation.

At the beginning of the edge-creation stage in day $t$, the interbank system comprises $N_{\mathrm{P}}$ isolated banks without any edges. Bank $i$ lends to bank $j$ on day $t$ with probability $p_{i j, t}$, 
given by

$$
p_{i j, t} \equiv \begin{cases}\left(a_{i, t} a_{j, t}\right)^{\alpha} & \text { if } i \notin B, j \notin L, \text { and }\{i, j\} \not \subset D, \\ 0 & \text { otherwise, }\end{cases}
$$

where $L, B$, and $D$ denote the sets of pure lenders, pure borrowers, and bidirectional traders, respectively. After applying this procedure to every combination of $(i, j)$, we remove all the edges and move on to day $t+1$. At the beginning of day $t+1$, the activity level of bank $i$ is updated as follows. With probability $h\left(a_{i, t}\right), a_{i, t+1}$ is reset to a random value drawn from the uniform distribution on $[0,1]$, where $h\left(a_{i}\right) \equiv c_{1}^{-1} a_{i}^{c_{2}}$. This reset probability is intended to capture the metabolism of the interbank market, in which some banks exit after continuous transactions while other banks enter after a long resting period. With probability $1-h\left(a_{i, t}\right)$, the activity level is updated according to a random walk process on the unit circle, given by

$$
\begin{aligned}
& a_{i, t+1}=\left|\cos \theta_{i, t+1}\right|, \\
& \theta_{i, t+1}=\theta_{i, t}+2 \pi \varepsilon_{i, t+1},
\end{aligned}
$$

where $\theta_{i, t+1}$ is a random-walk variable that describes the angle on the unit circle (see Fig. 4 for a schematic). Since an activity level must be on $[0,1], a_{i, t+1}$ is given by the absolute values of $\cos \theta_{i, t+1} . \varepsilon_{i, t+1}$ is a random variable uniformly distributed on $[-0.002,0.002]$. The introduction of fluctuations in $\varepsilon_{i}$ (and therefore in $\theta_{i}$ and $a_{i}$ ) would capture variations in money demand that banks are facing on a daily basis in practice. We note that assuming a random walk on the real line would not be able to replicate the empirical duration of bilateral transactions. The volatility of $\varepsilon$ is set such that the simulated bank turnover would well match the empirical one (Fig. S1). The initial value for the angle is set such that $\theta_{i, 0}=\arccos \left(a_{i, 0}\right)$, where $a_{i, 0}$ is drawn from the uniform distribution on $[0,1]$. The above two steps, edge creation and activity updating, are repeated until reaching the predefined terminal date $T$. Initial 5000 simulation periods are discarded to eliminate the influence of the initial conditions. We find that the combination $\left(\alpha, c_{1}, c_{2}\right)=(4,2000,2)$ gives the best fit to the empirical properties. The influence of parameters $c_{1}$ and $c_{2}$ on the slope of superlinear scaling is illustrated in Fig. S11.
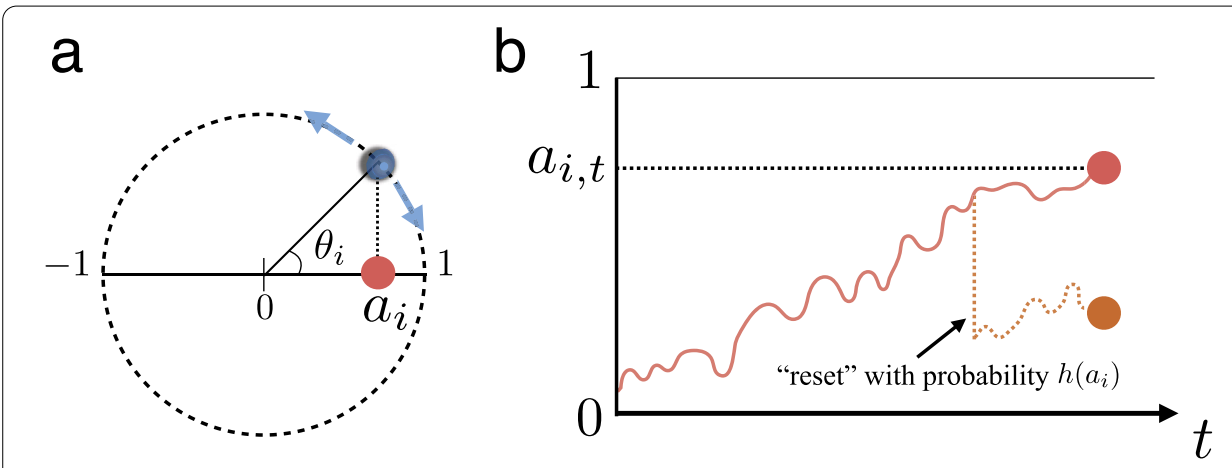

Figure 4 Schematic of the activity update in the dynamical fitness model. (a) Circular random walk of angle $\theta_{i}$ on the unit circle. Activity level of bank $i$ is given by $a_{i}=\left|\cos \theta_{i}\right|$. (b) Activity level $a_{i}$ evolves according to Eqs. (4) and (5) with reset probability $h\left(a_{i}\right)$ 


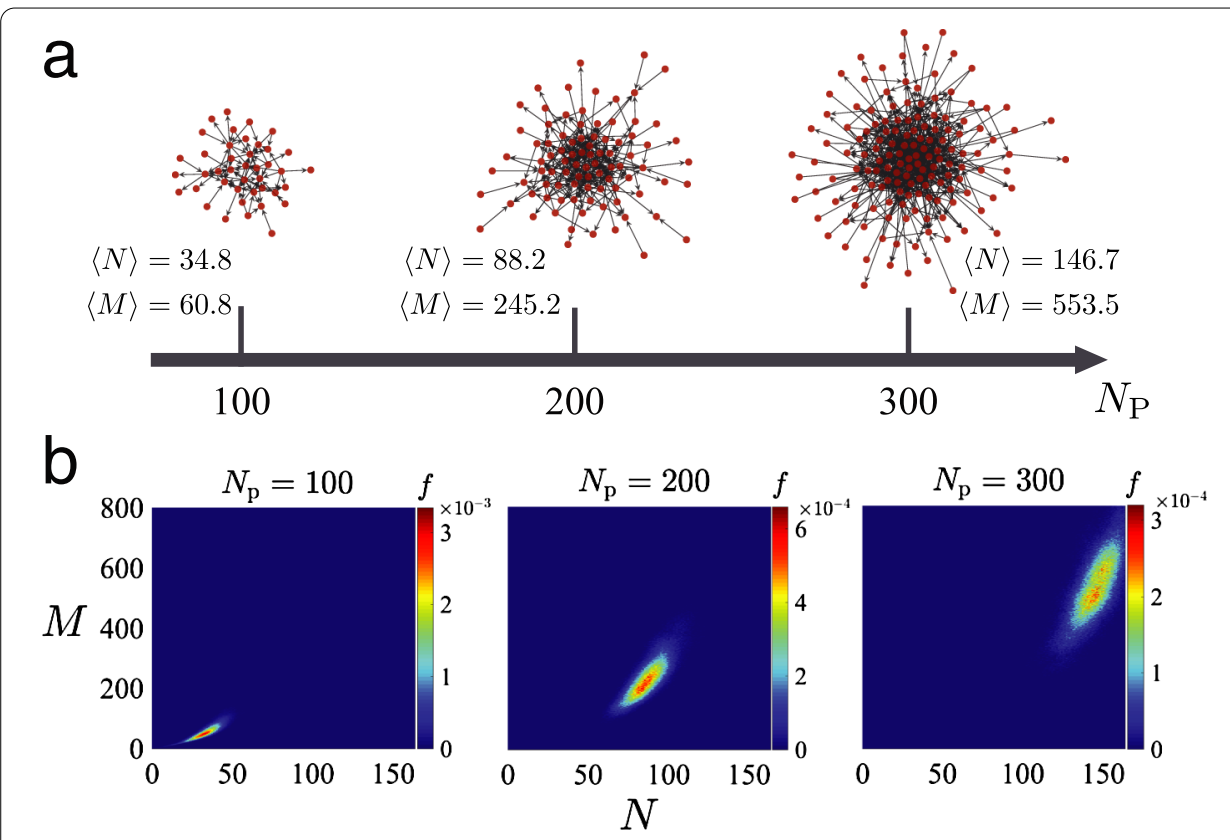

Figure 5 Generation of model networks. (a) Model networks with $N_{\mathrm{P}}=100,200$, and 300 (visualized by graph-tool [65]). The average values $\langle N\rangle$ and $\langle M\rangle$ for each $N_{\mathrm{P}}$ are also shown. (b) Joint conditional probability function $f\left(N, M \mid N_{P}\right)$ (color bar) for a given $N_{P}$

The simulated distributions of duration $\tau$ and interval $\Delta \tau$ for pairwise transactions replicate the empirical distributions for a given $N_{\mathrm{P}}$ (see lines in Fig. 2(b)-(c)). In addition, the growth pattern of the normalized aggregate degree $K(t) / K(T)$ is also successfully reproduced (Fig. 2(e)). We further evaluate the model fit for other dynamical properties such as the degree distribution and the strength as a function of degree [51] (Figs. S7-S9). We confirmed that the model can robustly reproduce the duration and interval distributions under different parameter settings (Figs. S4-S6).

\subsection{Fitting model to the data}

It should be noted that while the activity level $a_{i, t}$ fluctuates with time independently from other banks' activity levels, the model ensures that the size and the structure of generated networks are stationary for a given $N_{\mathrm{P}}$. In reality, however, the evolution of daily networks show a decreasing trend (Fig. 1(a) and (b)) and the network size varies from day to day owing to various external factors (e.g., shifts in monetary policy [39] and the seasonality of money demand due to the national holidays and/or the reserve requirement system [13]). In the model, the averages of the network size $\langle N\rangle$ and the number of edges $\langle M\rangle$ are controlled by tuning parameter $N_{\mathrm{P}}$ (Fig. 5(a)). Therefore, we need to estimate the daily sequence of $N_{\mathrm{P}}$ to reconstruct time series of empirical daily networks. Here, we take the following steps. First, we generate sufficiently many instances of synthetic networks for a given $N_{\mathrm{P}}$ to compute the histogram of $(N, M)$ (Fig. 5(b)). Generating networks over a sufficiently broad range of $N_{\mathrm{P}}$ provides a conditional probability function $f\left(N, M \mid N_{\mathrm{P}}\right)$ that would cover the range of $N$ and $M$ observed in the empirical networks (Fig. S10). Second, for a given empirical daily network with $\left(N^{\prime}, M^{\prime}\right)$, we choose a daily estimate of $N_{\mathrm{P}}$, denoted by $N_{\mathrm{P}, \mathrm{ML}}$, such that $N_{\mathrm{P}, \mathrm{ML}}=\operatorname{argmax}_{N_{\mathrm{P}}} f\left(N^{\prime}, M^{\prime} \mid N_{\mathrm{P}}\right)$. 


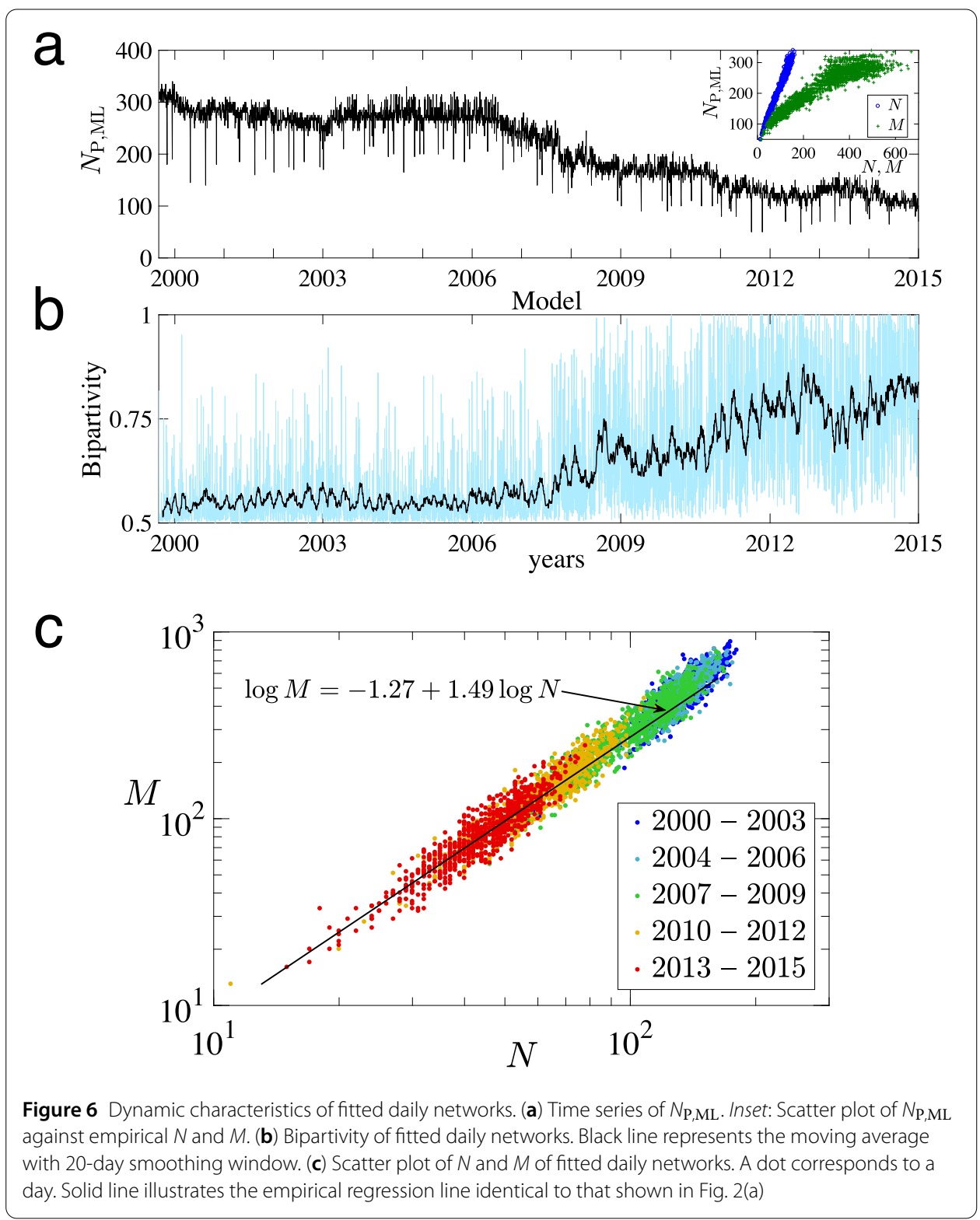

We find that simulated instances of $(N, M)$ concentrate tightly along the regression line of $M \propto N^{1.5}$ when we vary $N_{\mathrm{P}}$ from 50 to 350 (Fig. S10). This agreement holds true even under alternative parameter values within a reasonable range of variation (Figs. S4(a) and S11). The sequence of daily estimates of $N_{\mathrm{P}, \mathrm{ML}}$, each of which is based on the empirical combination $\left(N^{\prime}, M^{\prime}\right)$ of a day, exhibit a long-term downward trend consistent with the empirical sequence of $N$ and $M$ (Fig. 6(a)). Specifically, $N_{\mathrm{P}, \mathrm{ML}}$ is proportional to $N$ and increases nonlinearly in $M$ with saturation at $N_{\mathrm{P}, \mathrm{ML}} \approx 300$ (Fig. 6(a), Inset).

A time series of model networks based on the daily estimates of $N_{\mathrm{P}, \mathrm{ML}}$ reproduces the tendency toward a perfect bipartite structure (Fig. 6(b)), although we have not explicitly modeled how the network structure should change with $N_{\mathrm{p}}$. The tendency toward a bipartite structure may reflect the fact that the average degree of generated networks becomes smaller as the size of network shrinks. Indeed, the relationship between $N$ and $M$ across 
the fitted daily networks explains the emergence of superlinearity (Fig. 6(c)), which indicates that $M \propto N^{1.5}$ or $\langle k\rangle \propto \sqrt{N}$.

\section{Discussion}

The time series of daily networks reveal many dynamical regularities encoded in millions of financial transactions. An important finding is that the transaction patterns between banks are similar to the social communication patters of humans, which have been observed at higher temporal resolutions (typically $20 \sim 60$ seconds) than a daily resolution. For instance, a power-law scaling in the distribution of the interaction duration has been found in human contact networks, such as face-to-face conversation networks of individuals [36, 37]. We note that the general economic meaning of long-term relationships between a lender (e.g., a bank) and a borrower (e.g., a firm) has long been studied in microeconomics, and some studies pointed out that there also exist long-term relationships between banks in interbank markets $[28,52-55]$. This would imply that there could be common mechanisms that govern social behavior of humans and economic behavior of financial institutions. Although the social activity patterns have been found only in social systems so far, given our findings, it would be reasonable to speculate that similar activity patterns might be found in other complex systems as well as in financial systems.

The sublinear growth pattern of aggregated degree has also been reported in the mobility pattern of mobile-phone users [47]. In addition, superlinear scaling at the network level (called "urban scaling" [40]) emerges in various social contexts, such as the relationship between the number of mobile connections and the population size of cities [38]. The discovered dynamical patterns are quite robust and hold true even amid the global financial crisis, suggesting that there is a universal mechanism connecting financial and social dynamics.

The contribution of our work is not limited to the findings on the transition patterns of interbank networks. The model we propose here can be used as a generator of synthetic networks for studies of financial systemic risk. As is often the case, inaccessibility to empirical data on financial transactions forces academic researchers to use synthetic networks with limited empirical properties $[15,25]$ or to infer real network structure based on available partial information $[18,56-62]$. Our model provides a way to easily generate synthetic time series of networks that exhibit dynamical properties characterizing the daily evolution of real interbank networks. We hope that the current work will not only deepen our knowledge about the dynamic nature of interbank networks, but also help to improve the conventional approach of systemic-risk studies toward a more dynamic analysis.

We leave three remaining issues that need to be addressed in future research. First, while the observed dynamical patterns are quite robust and seem universal given the similarity to social network dynamics, it is worth investigating whether those findings hold true in other countries as well. Second, we might be able to find other dynamical patterns at different time resolutions such as intraday, weekly, and monthly. If that is the case, we need to see if those dynamical patterns found in different time scales are consistently explained by the current model. Finally, our finding reveals an independence of local interaction patterns of banks from a global-scale network evolution, such as the decreasing trend in network size. This implies that there is no feedback loop between micro and macroscopic phenomena, meaning that banks are not adaptive to their environments. Further research is needed to explain why such a decoupling phenomenon takes hold in financial networks. 


\section{Methods}

\subsection{Data description}

The original time-stamped data are commercially available from e-MID SIM S.p.A based in Milan, Italy [35]. The data contain all the unsecured euro-denominated transactions between financial institutions made via an online trading platform, e-MID. We focus on overnight (labelled as "ON") and overnight-large ("ONL") transactions. ON transactions refer to contracts that require borrowers to repay the full amount within one business day from the day the loans are executed. ONL transactions are a variant of the ON transactions, where the amount is no less than 100 million euros.

The data processing procedure is as follows. First, we extract transactions made between September 4, 2000 and December 31, 2015. The choice of the initial date is based on the introduction of the ONL category [13]. This leaves us with 1,119,258 ON and 73,480 ONL transactions, which comprise $86 \%$ of all the transactions during that period. Next, we transform all the ON and ONL transactions into a sequence of daily networks by applying the daily time window of 8:00-18:00 [28]. We then extract the transactions that belong to the largest weakly connected component of each daily network, which account for $99.3 \%$ of all the daily transactions on average (the minimum is 78\%). We referred to this component as daily network throughout the analysis. Multiple edges between two banks are simplified. In the end, we have 1,187,415 transactions conducted by 308 financial institutions over 3922 business days.

\subsection{Fitting procedure for the interval distribution}

As shown in Fig. 2(c), the empirical distribution of transaction interval $\Delta \tau$ for each bank pair does not follow a power law. We instead find that the interval distribution nicely fits a Weibull distribution for $1 \leq \Delta \tau<\Delta \tau_{\mathrm{u}}$, where $\Delta \tau_{\mathrm{u}}$ denotes a cutoff value.

The complementary cumulative distribution function (CCDF) of a Weibull distribution [46] is given by

$$
P_{c}(x)=\exp \left\{-\left(\frac{x}{\lambda}\right)^{c}\right\} \text { for } x>0
$$

where $c>0$ and $\lambda>0$ are parameters. Distribution $P_{c}(x)$ can also be written as $n_{x} / N_{X}$, where $N_{X}$ is the total number of interval values observed, and $n_{x}$ is the rank of interval length $x$ (i.e., $n_{x}$ is the number of observed interval values such that $\Delta \tau \geq x$ ). By taking the logarithm of $n_{x} / N_{X}=\exp \left\{-(x / \lambda)^{c}\right\}$, we obtain the following expression [45]:

$$
\left(x_{n}\right)^{c}=-\beta\left(\log n_{x}-\log N_{X}\right),
$$

where $x_{n}$ represents the interval length whose rank is $n$ (i.e., $x_{1}>x_{2}>\cdots>x_{N_{X}}$ ), and $\beta$ is defined as $\beta \equiv \lambda^{c}$. We use Eq. (7) to find $\beta$ and $c$ that give the best fit to a Weibull distribution. We introduce $\hat{n}$, the logged rank of cutoff value $\Delta \tau_{u}$, and estimate parameters $(\beta, c)$ for a subset of the observed values of $\Delta \tau$, in a similar way as is done in the standard estimation procedure for a power-law exponent [43]. The cutoff $\Delta \tau_{\mathrm{u}}$ corresponds to the $e^{\hat{n}}$ th largest interval length. Parameters $\beta, c$, and $\hat{n}$ are determined as follows.

1. For a given pair $(c, \hat{n})$, estimate $\beta$ in (7) by the ordinary least squares (OLS). Repeat this for sufficiently many values of $c \in[0,1)$ (we set $c<1$ because the tail of the 

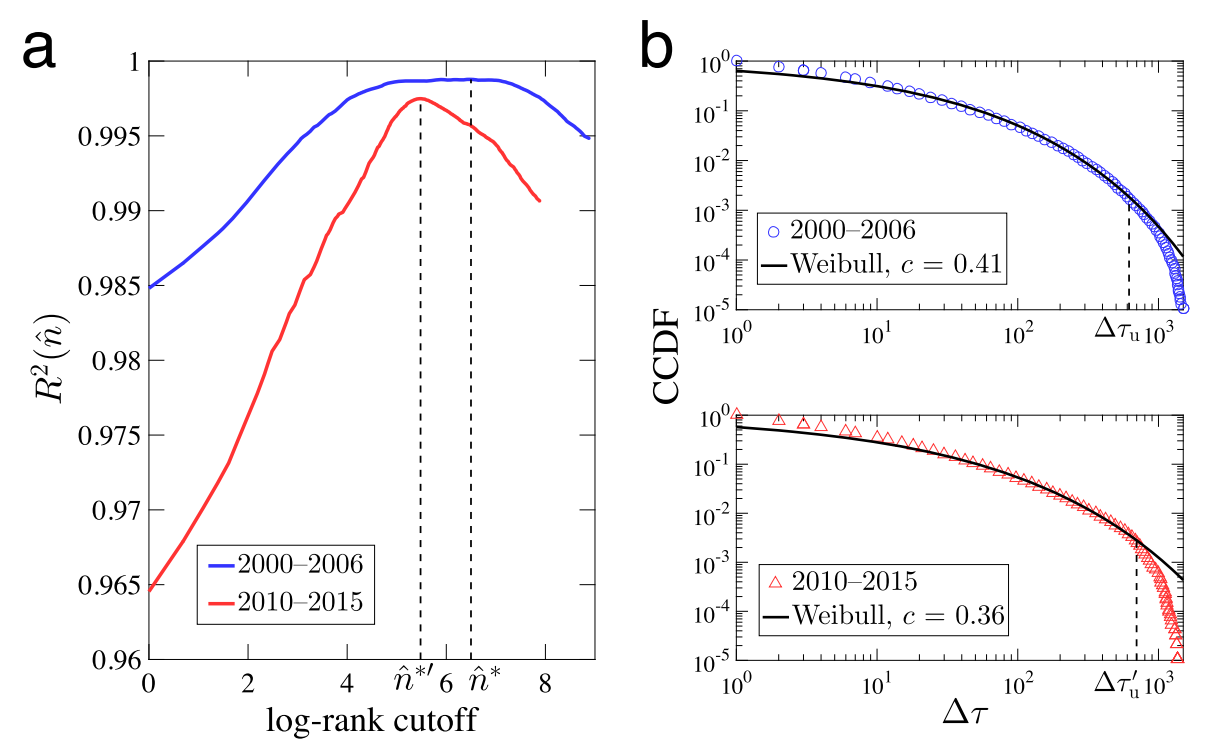

Figure 7 Fitting of the interval distribution with a Weibull distribution. (a) Determination of the optimal log-rank cutoff $\hat{n}^{*}$. (b) Empirical CCDF of interval $\Delta \tau$ (symbols) and the Weibull distribution with the estimated parameters (lines). The cutoffs $\Delta \tau_{u}$ and $\Delta \tau_{u}^{\prime}$ are obtained from the optimal log-rank cutoffs $\hat{n}^{*}$ and $\hat{n}^{* \prime}$, respectively

empirical distribution of $\Delta \tau$ is apparently heavier than that of an exponential distribution). The estimate of $\beta$ is denoted by $\beta^{*}(c, \hat{n})$.

2. For $\hat{n}$ given in step 1 , find the optimal value of $c$, denoted by $c^{*}(\hat{n})$, such that the coefficient of determination $R^{2}$ for the OLS regression is maximized, in which case $\beta=\beta^{*}\left(c^{*}(\hat{n}), \hat{n}\right)$. Let $R^{2}(\hat{n})$ denote the maximum of $R^{2}$ for a given $\hat{n}$.

3. By repeating steps 1 and 2 for all the predefined values of $\hat{n}$, find the optimal cutoff value $\hat{n}^{*} \equiv \operatorname{argmax}_{\hat{n}} R^{2}(\hat{n})$. In the end, the estimates of the parameters are given by $\hat{n}=\hat{n}^{*}, c=c^{*}\left(\hat{n}^{*}\right)$, and $\beta=\beta^{*}\left(c^{*}\left(\hat{n}^{*}\right), \hat{n}^{*}\right)$.

Figure 7(a) illustrates the determination of the optimal log-rank cutoff $\hat{n}^{*}$. The inset of Fig. 2(c) in the main text shows the OLS fit to (7) when $\hat{n}=\hat{n}^{*}$ (note that $x_{n}$ corresponds to $\Delta \tau$ in that figure). Once $\hat{n}^{*}$ is determined, it is straightforward to obtain the corresponding cutoff $\Delta \tau_{u}$. Figure $7(\mathrm{~b})$ verifies the goodness of fit between the empirical CCDF and the estimated Weibull distribution.

\subsection{Analytical solution for the fitness model with a finite size effect}

Relationship between $N$ and $M$. As we described in the main text, we assume that initially there are $N_{\mathrm{P}}$ many isolated nodes. Node $i\left(1 \leq i \leq N_{\mathrm{P}}\right)$ is assigned a fitness $a_{i} \in[0,1]$ which is drawn from density $\rho(a)$.

The probability of edge formation between two nodes $i$ and $j$ is denoted by $u\left(a_{i}, a_{j}\right)$. We define $N$ as the number of nodes connected with at least one edge and $M$ as the total number of edges in a network. We express $N$ and $M$ as functions of $N_{\mathrm{P}}$ :

$$
\left\{\begin{array}{l}
N=\left(1-q_{0}\left(N_{\mathrm{P}}\right)\right) N_{\mathrm{P}}, \\
M=\frac{\bar{k}\left(N_{\mathrm{P}}\right) N_{\mathrm{P}}}{2},
\end{array}\right.
$$


where $q_{0}\left(N_{\mathrm{P}}\right)$ is the probability of a randomly chosen node being isolated (i.e., no edges attached) and $\bar{k}\left(N_{\mathrm{P}}\right)$ is the average degree over all nodes including isolated ones. Thus, to obtain the functional forms of $N$ and $M$, we need to get the functional forms of $q_{0}\left(N_{\mathrm{P}}\right)$ and $\bar{k}\left(N_{\mathrm{P}}\right)$. In the following, we first derive the functional forms of $q_{0}\left(N_{\mathrm{P}}\right)$ and $\bar{k}\left(N_{\mathrm{P}}\right)$ in a general setting. Then, we will restrict our attention to the case with $\rho(a)=1$ (i.e., a uniform distribution) and $u\left(a_{i}, a_{j}\right)=\left(a_{i} a_{j}\right)^{\alpha}$ to explain the empirical superlinear relation between $N$ and $M$ in the same specification as in the main text.

Average degree of networks including isolated nodes, $\bar{k}\left(N_{\mathrm{P}}\right)$. Given the fitnesses of all nodes $\boldsymbol{a}=\left(a_{1}, a_{2}, \ldots, a_{N_{\mathrm{P}}}\right)$, the probability that node $i$ has degree $k_{i}$ is

$$
g\left(k_{i} \mid \boldsymbol{a}\right)=\sum_{c_{i}}\left[\prod_{j \neq i} u\left(a_{i}, a_{j}\right)^{c_{i j}}\left(1-u\left(a_{i}, a_{j}\right)\right)^{1-c_{i j}}\right] \times \delta\left(\sum_{j \neq i} c_{i j}, k_{i}\right)
$$

where $c_{i j} \in\{0,1\}$ is the $(i, j)$-element of the adjacency matrix and $\boldsymbol{c}_{i}=\left(c_{1 i}, c_{2 i}, \ldots, c_{N_{\mathrm{P}}}\right)^{\top}$ is the $i$ th column vector. Function $\delta(x, y)$ denotes the Kronecker delta. Let us redefine a product term in the square bracket of (9) as

$$
f_{j}\left(c_{i j} ; a_{i}, a_{j}\right) \equiv u\left(a_{i}, a_{j}\right)^{c_{i j}}\left(1-u\left(a_{i}, a_{j}\right)\right)^{1-c_{i j}} .
$$

Since $g\left(k_{i} \mid \boldsymbol{a}\right)$ is the convolution of $\left\{f_{j}\left(c_{i j} ; a_{i}, a_{j}\right)\right\}_{j}$, its generating function

$$
\hat{g}_{i}(z \mid \boldsymbol{a}) \equiv \sum_{k_{i}} z^{k_{i}} g\left(k_{i} \mid \boldsymbol{a}\right)
$$

is decomposed as

$$
\hat{g}_{i}(z \mid \boldsymbol{a})=\prod_{j \neq i} \hat{f}_{j}\left(z ; a_{i}, a_{j}\right)
$$

where $\hat{f}_{j}$ is the generating function of $f_{j}\left(c_{i j} ; a_{i}, a_{j}\right)$, given by

$$
\hat{f}_{j}\left(z ; a_{i}, a_{j}\right) \equiv \sum_{a_{i j}} z^{a_{i j}} f_{j}\left(a_{i j} ; a_{i}, a_{j}\right)
$$

Degree distribution $p\left(k_{i} ; N_{\mathrm{P}}\right)$ is defined by the probability that node $i$ has degree $k_{i}$ and is related to $g\left(k_{i} \mid \boldsymbol{a}\right)$ such that

$$
p\left(k_{i} ; N_{\mathrm{P}}\right)=\int g\left(k_{i} \mid \boldsymbol{a}\right) \rho(\boldsymbol{a}) d \boldsymbol{a}
$$

where we define $\rho(\boldsymbol{a}) \equiv \prod_{i} \rho\left(a_{i}\right)$ and $d \boldsymbol{a} \equiv \prod_{i} d a_{i}$. Therefore, differentiation of $\hat{g}_{i}(z \mid \boldsymbol{a})$ with respect to $z$ gives the average degree $\bar{k}\left(N_{\mathrm{P}}\right)$ :

$$
\begin{aligned}
\bar{k}\left(N_{\mathrm{P}}\right) & =\sum_{k_{i}} k_{i} p\left(k_{i} ; N_{\mathrm{P}}\right) \\
& =\sum_{k_{i}} k_{i} \int g\left(k_{i} \mid \boldsymbol{a}\right) \rho(\boldsymbol{a}) d \boldsymbol{a}
\end{aligned}
$$




$$
\begin{aligned}
& =\left.\frac{d}{d z} \int \hat{g}_{i}(z \mid \boldsymbol{a}) \rho(\boldsymbol{a}) d \boldsymbol{a}\right|_{z=1} \\
& =\left.\frac{d}{d z} \int \rho\left(a_{i}\right) d a_{i} \prod_{j \neq i} \int \hat{f}_{j}\left(z ; a_{i}, a_{j}\right) \rho\left(a_{j}\right) d a_{j}\right|_{z=1} \\
& =\left.\int \rho\left(a_{i}\right) d a_{i} \frac{d}{d z}\left[\int \hat{f}\left(z ; a_{i}, h\right) \rho(h) d h\right]^{N_{\mathrm{P}}-1}\right|_{z=1} \\
& =\left.\left(N_{\mathrm{P}}-1\right) \int \rho\left(a_{i}\right) d a_{i}\left[\int d a \rho(a) \hat{f}\left(z ; a_{i}, a\right)\right]^{N_{\mathrm{P}}-2} \int d a \rho(a) \frac{d}{d z} \hat{f}\left(z ; a_{i}, a\right)\right|_{z=1} .
\end{aligned}
$$

From Eqs. (10) and (13), we have $\hat{f}\left(z ; a_{i}, a\right)=\sum_{c_{i j}} z^{c_{i j}} f\left(c_{i j} ; a_{i}, a\right)=(z-1) u\left(a_{i}, a\right)+1$. It follows that

$$
\begin{aligned}
& \int d a \rho(a) \hat{f}\left(z ; a_{i}, a\right)=(z-1) \int d a \rho(a) u\left(a_{i}, a\right)+1, \\
& \int d a \rho(a) \frac{d}{d z} \hat{f}\left(z ; a_{i}, a\right)=\int d a \rho(a) u\left(a_{i}, a\right) .
\end{aligned}
$$

Substituting these into Eq. (15) leads to

$$
\bar{k}\left(N_{\mathrm{P}}\right)=\left(N_{\mathrm{P}}-1\right) \iint d a d a^{\prime} \rho(a) \rho\left(a^{\prime}\right) u\left(a, a^{\prime}\right) .
$$

It should be noted that (18) is equivalent to Eq. (21) of Ref. [50].

Probability of node isolation, $q_{0}\left(N_{\mathrm{P}}\right)$. From (14), the probability of a node being isolated, $q_{0}\left(N_{\mathrm{P}}\right) \equiv p\left(k_{i}=0 ; N_{\mathrm{P}}\right)$, is given by

$$
\begin{aligned}
q_{0}\left(N_{\mathrm{P}}\right) & =\int g\left(k_{i}=0 \mid \boldsymbol{a}\right) \rho(\boldsymbol{a}) d \boldsymbol{a} \\
& =\int d a_{i} \rho\left(a_{i}\right)\left[1-\int u\left(a_{i}, a\right) \rho(a) d a\right]^{N_{\mathrm{P}}-1} .
\end{aligned}
$$

Special case: $\rho(a)=1$ and $u\left(a, a^{\prime}\right)=\left(a a^{\prime}\right)^{\alpha}$. Substituting $\rho(a)=1$ and $u\left(a, a^{\prime}\right)=\left(a a^{\prime}\right)^{\alpha}$ into Eq. (18) gives

$$
\bar{k}\left(N_{\mathrm{P}}\right)=\left(\frac{1}{\alpha+1}\right)^{2}\left(N_{\mathrm{P}}-1\right) .
$$

Similarly, substituting the same conditions into Eq. (19) gives

$$
q_{0}\left(N_{\mathrm{P}}\right)=\int d a_{i}\left(1-\frac{1}{\alpha+1} a_{i}^{\alpha}\right)^{N_{\mathrm{P}}-1} .
$$

By rewriting the integrand as $x=1-\frac{1}{\alpha+1} a_{i}^{\alpha}$, we have

$$
\begin{aligned}
q_{0}\left(N_{\mathrm{P}}\right) & =\frac{1}{\alpha}(\alpha+1)^{\frac{1}{\alpha}} \int_{1-\frac{1}{\alpha+1}}^{1}(1-x)^{\frac{1}{\alpha}-1} x^{N_{\mathrm{P}}-1} d x \\
& =\frac{1}{\alpha}(\alpha+1)^{\frac{1}{\alpha}}\left[\mathrm{B}\left(N_{\mathrm{P}}, \frac{1}{\alpha}\right)-\mathrm{B}_{1-\frac{1}{\alpha+1}}\left(N_{\mathrm{P}}, \frac{1}{\alpha}\right)\right],
\end{aligned}
$$


where $\mathrm{B}(x, y) \equiv \int_{0}^{1} t^{x-1}(1-t)^{y-1} d t$ is the beta function and $\mathrm{B}_{z}(x, y) \equiv \int_{0}^{z} t^{x-1}(1-t)^{y-1} d t$ $(0<\operatorname{Re}(z) \leq 1)$ is the incomplete beta function. Combining these results with Eq. (8), we end up with

$$
\left\{\begin{array}{l}
N=N_{\mathrm{P}}\left[1-\frac{1}{\alpha}(\alpha+1)^{\frac{1}{\alpha}}\left(\mathrm{B}\left(N_{\mathrm{P}}, \frac{1}{\alpha}\right)-\mathrm{B}_{1-\frac{1}{\alpha+1}}\left(N_{\mathrm{P}}, \frac{1}{\alpha}\right)\right)\right], \\
M=\left(\frac{1}{\alpha+1}\right)^{2} \frac{N_{\mathrm{P}}\left(N_{\mathrm{P}}-1\right)}{2} .
\end{array}\right.
$$

If $N_{\mathrm{P}}$ is sufficiently large, then $q_{0}\left(N_{\mathrm{P}}\right) \simeq 0$ and thereby $N \simeq N_{\mathrm{P}}$ and $M \simeq(1 / \alpha+1)^{2} N(N-$ 1) $/ 2 \propto N^{2}$. Therefore, the solution is consistent with that of the previous studies [48-50] in the absence of the finite-size effect.

\subsection{Dynamics of weights}

Empirical observation. On top of the edge dynamics that we discussed in the main text, the dynamics of edge weights also exhibits specific patterns. Let us define the weight of an edge, $w_{i j, t}$, as the total amount of funds transferred from bank $i$ to $j$ on day $t$. We define the growth rate of edge weights as $r_{i j, t} \equiv \log \left(w_{i j, t+1} / w_{i j, t}\right)$ for bank pair $(i, j)$ such that $w_{i j, t+1} w_{i j, t}>$ 0 [51]. The distribution of $r_{i j, t}$, aggregated over all pairs and all $t$, exhibits a symmetric triangular shape with a distinct peak at 0 (Fig. 8(a)). The shape of the distribution indicates that a large fraction of bank pairs do not change the amount of funds when they keep trading, and if they change the amount, the rate of change will be typically small. A similar sort of triangular-shaped distribution of the growth rate of weights has also been found in networks of email exchanges [63], airlines [51] and cattle trades between stock farming facilities [64].

Model of weight dynamics. To reproduce the dynamics of edge weights, we add the following step to the model. Let us consider the edge between $i$ and $j$ formed in day $t$. If there is an edge from $i$ to $j$ in day $t-1$, then the edge weights in day $t$ is given by

$$
w_{i j, t} \equiv \begin{cases}w_{i j, t-1} & \text { with probability } 1-q, \\ \kappa v_{i j, t} p_{i j, t} & \text { with probability } q,\end{cases}
$$

where random variable $v_{i j, t}$ takes different values across bank pairs and are assumed to follow a power-law distribution with exponent $\eta$ to maximize the fit to $P(r)$ (Fig. 8) and
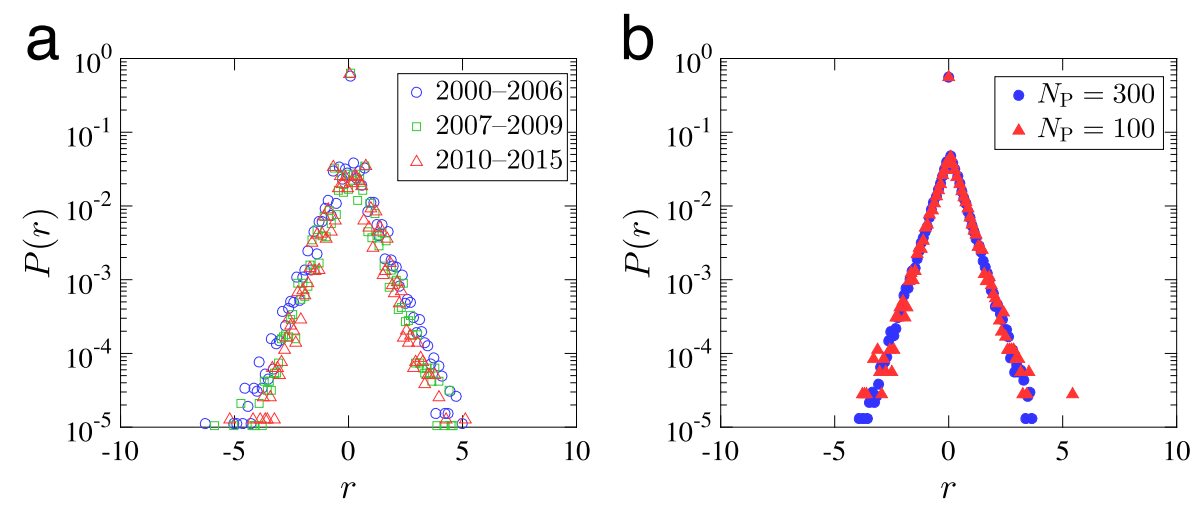

Figure 8 Distribution of the growth rates of edge weights $r$ (aggregated over all bank pairs) for (a) the data and (b) the model 

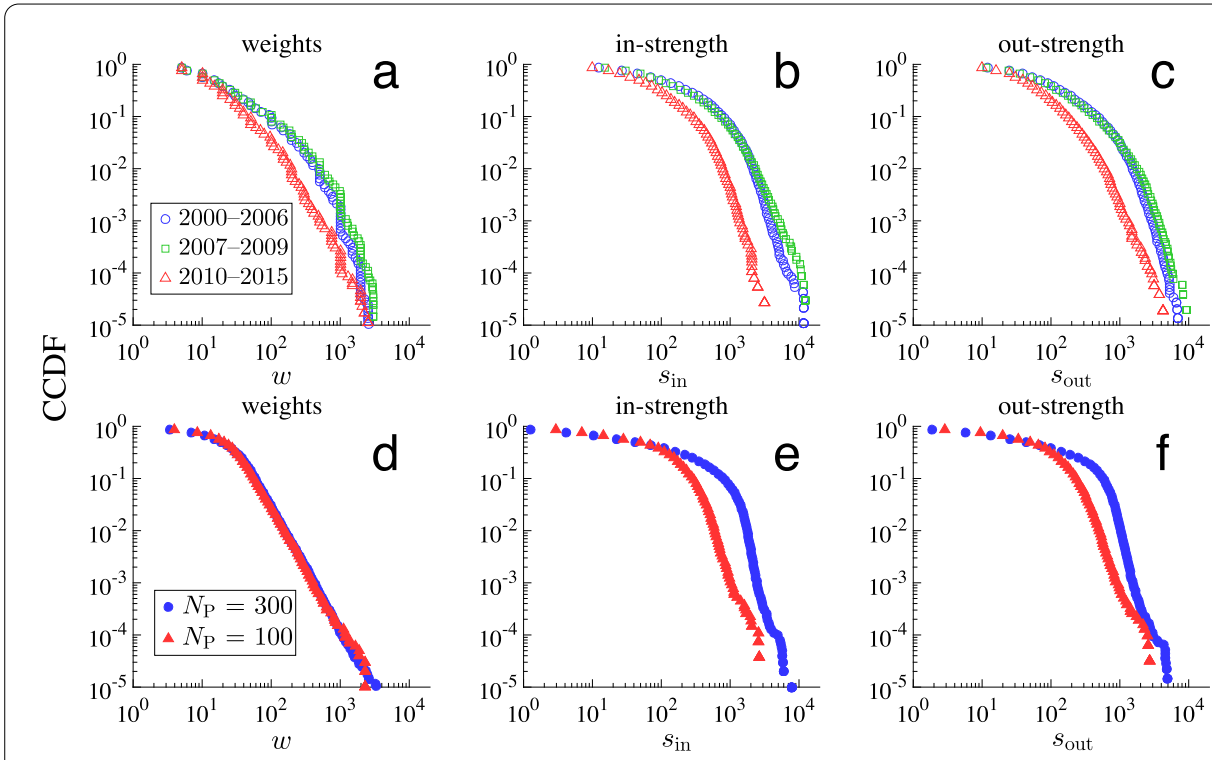

Figure 9 CCDF of edge weights and strength. The in- and out-strength of bank $i$ are defined as $s_{i n, i}=\sum_{j \neq i} w_{j i}$ and $s_{\text {out }, i}=\sum_{j \neq i} w_{i j}$, respectively. (a)-(c) The data. (d)-(f) The model

the empirical weight distribution (Fig. 9(a)-(c)). Positive constant $\kappa$ is introduced to match the scale of edge weights with that of the data (i.e., millions of euros). On the other hand, if there is no edge from $i$ to $j$ in day $t-1$ but there is in day $t$, then

$$
w_{i j, t} \equiv \kappa v_{i j, t} p_{i j, t} .
$$

Any non-adjacent pairs $(i, j)$ has $w_{i j, t}=0$.

We set the weight parameters as $(q, \kappa, \eta)=(0.5,80,3.3)$ to fit $P(r)$ and the simulated weight distributions with the empirical ones, respectively. Figures $8(b)$ and $9(d)-(f)$ show that our model of weight dynamics successfully replicates the empirical distributions.

\section{Acknowledgements}

The authors thank Naoki Masuda for useful comments on the manuscript and Research Project "Network Science" organized at International Institute for Advanced Studies for providing an opportunity to initiate the project.

\section{Funding}

TK acknowledges financial support from the Japan Society for the Promotion of Science Grants no. $15 \mathrm{H0} 05729$ and 16K03551. TT was supported by JST ERATO Grant Number JPMJER1201, Japan.

Availability of data and materials

The original data are commercially available from e-MID SIM S.p.A based in Milan, Italy [35]. MATLAB code for generating synthetic networks is available from Teruyoshi Kobayashi's website: https://terukobayashi.wordpress.com/.

\section{Competing interests}

The authors declare that they have no competing financial interests.

\section{Authors' contributions}

TK and TT conceived the research. TK conducted the empirical and numerical analysis. TT processed the data and conducted the theoretical analysis. TK and TT discussed the results and wrote the manuscript. All authors read and approved the final manuscript.

\section{Author details}

${ }^{1}$ Graduate School of Economics, Kobe University, Kobe, Japan. ${ }^{2}$ National Institute of Information and Communications Technology, Tokyo, Japan. ${ }^{3}$ National Institute of Informatics, Tokyo, Japan. ${ }^{4}$ JST, ERATO, Kawarabayashi Large Graph Project, Tokyo, Japan. 


\section{Publisher's Note}

Springer Nature remains neutral with regard to jurisdictional claims in published maps and institutional affiliations.

\section{Received: 16 October 2017 Accepted: 29 May 2018 Published online: 07 June 2018}

\section{References}

1. Brunnermeier MK (2009) Deciphering the liquidity and credit crunch 2007-2008. J Econ Perspect 23:77-100

2. Diamond DW, Rajan RG (2009) Fear of fire sales and the credit freeze. NBER W, 14925

3. Allen F, Carletti E (2010) An overview of the crisis: causes, consequences, and solutions. Int Rev Finance 10:1-26

4. Anand K, Gai P, Marsili M (2012) Rollover risk, network structure and systemic financial crises. J Econ Dyn Control 36:1088-1100

5. Acharya WV, Merrouche $O$ (2013) Precautionary hoarding of liquidity and interbank markets: evidence from the subprime crisis. Rev Finance 17:107-160

6. Cimini G, Serri M (2016) Entangling credit and funding shocks in interbank markets. PLoS ONE 11:e0161642

7. Mishkin FS (2011) Monetary policy strategy: lessons from the crisis. NBER W, 16755

8. Atkinson T, Luttrell D, Rosenblum H (2013) How bad is it? The costs and consequences of the 2007-09 financial crisis. Dallas Fed Staff Papers 20

9. May RM, Levin SA, Sugihara G (2008) Complex systems: ecology for bankers. Nature 451:893-895

10. Schweitzer F et al (2009) Economic networks: the new challenges. Science 325:422-425

11. Helbing D (2013) Globally networked risks and how to respond. Nature 497:51-59

12. Battiston $S$ et al (2016) Complexity theory and financial regulation. Science 351:818-819

13. Beaupain R, Durré A (2008) The interday and intraday patterns of the overnight market: Evidence from an electronic platform. ECB W, 988

14. Cifuentes R, Ferrucci G, Shin HS (2005) Liquidity risk and contagion. J Eur Econ Assoc 3:556-566

15. Huang X, Vodenska I, Havlin S, Stanley HE (2013) Cascading failures in bi-partite graphs: model for systemic risk propagation. Sci Rep 3:1219

16. Upper C, Worms A (2004) Estimating bilateral exposures in the German interbank market: is there a danger of contagion? Eur Econ Rev 48:827-849

17. Elsinger H, Lehar A, Summer M (2006) Risk assessment for banking systems. Manag Sci 52:1301-1314

18. Lelyveld IV, Liedorp F (2006) Interbank contagion in the Dutch banking sector: a sensitivity analysis. Int J Cent Bank 2:99-133

19. Cont R, Moussa A, Santos EB (2013) Network structure and systemic risk in banking systems in handbook on systemic risk. In: Fouque JP, Langsam JA (eds) Handbook on Systemic Risk. Cambridge University Press, New York.

20. Bardoscia M, Battiston S, Caccioli F, Caldarelli G (2017) Pathways towards instability in financial networks. Nat Commun 8:14416

21. Nier E, Yang J, Yorulmazer T, Alentorn A (2007) Network models and financial stability. J Econ Dyn Control 31:2033-2060

22. Gai P, Kapadia S (2010) Contagion in financial networks. Proc R Soc Lond Ser A, Math Phys Sci 466:2401-2423

23. Haldane AG, May RM (2011) Systemic risk in banking ecosystems. Nature 469:351-355

24. Tedeschi G, Mazloumian A, Gallegati M, Helbing D (2013) Bankruptcy cascades in interbank markets. PLoS ONE 7:e52749

25. Brummitt CD, Kobayashi T (2015) Cascades in multiplex financial networks with debts of different seniority. Phys Rev E 91:062813

26. Burkholz R, Leduc MV, Garas A, Schweitzer F (2016) Systemic risk in multiplex networks with asymmetric coupling and threshold feedback. Physica D 323:64-72

27. Caccioli F, Farmer JD, Foti N, Rockmore D (2015) Overlapping portfolios, contagion, and financial stability. J Econ Dyn Control 51:50-63

28. Iori G et al (2015) Networked relationships in the e-MID interbank market: a trading model with memory. J Econ Dyn Control 50:98-116

29. Finger K, Fricke D, Lux T (2013) Network analysis of the e-MID overnight money market: the informational value of different aggregation levels for intrinsic dynamic processes. Comput Manag Sci 10:187-211

30. Battiston S, Caldarelli G, May RM, Roukny T, Stiglitz JE (2016) The price of complexity in financial networks. Proc Natl Acad Sci USA 113:10031-10036

31. Boss M, Elsinger H, Summer M, Thurner S (2004) Network topology of the interbank market. Quant Finance 4:677-684

32. Soramäki K, Bech ML, Arnold J, Glass RJ, Beyeler WE (2007) The topology of interbank payment flows. Physica A 379:317-333

33. Iori G, De Masi G, Precup OV, Gabbi G, Caldarelli G (2008) A network analysis of the Italian overnight money market. J Econ Dyn Control 32:259-278

34. Imakubo K, Soejima Y (2010) The transaction network in Japan's interbank money markets. Bank Japan Monet Econ Stud 28:107-150

35. http://www.e-mid.it/

36. Cattuto C et al (2010) Dynamics of person-to-person interactions from distributed RFID sensor networks. PLoS ONE 5:e11596

37. Starnini M, Baronchelli A, Pastor-Satorras R (2013) Modeling human dynamics of face-to-face interaction networks. Phys Rev Lett 110:168701

38. Schläpfer M et al (2014) The scaling of human interactions with city size. J R Soc Interface 11:20130789

39. Barucca P, Lillo F (2018) The organization of the interbank network and how ECB unconventional measures affected the e-MID overnight market. Comput Manag Sci 15:33-53

40. Bettencourt LM, Lobo J, Helbing D, Kühnert C, West GB (2007) Growth, innovation, scaling, and the pace of life in cities. Proc Natl Acad Sci USA 104:7301-7306

41. Bettencourt LM (2013) The origins of scaling in cities. Science 340:1438-1441 
42. Pan W, Ghoshal G, Krumme C, Cebrian M, Pentland A (2013) Urban characteristics attributable to density-driven tie formation. Nat Commun 4:1961

43. Clauset A, Shalizi CR, Newman MEJ (2009) Power-law distributions in empirical data. SIAM Rev 51:661-703

44. http://tuvalu.santafe.edu/ aaronc/powerlaws/

45. Sornette D (2006) Critical phenomena in natural sciences: chaos, fractals, selforganization and disorder: concepts and tools. Springer, Berlin

46. Weibull W (1951) A statistical distribution of wide applicability. J Appl Mech 103:293-297

47. Song C, Koren T, Wang P, Barabási AL (2010) Modelling the scaling properties of human mobility. Nat Phys 6:818-823

48. Caldarelli G, Capocci A, De Los Rios P, Muñoz MA (2002) Scale-free networks from varying vertex intrinsic fitness. Phys Rev Lett 89:258702

49. De Masi G, lori G, Caldarelli G (2006) Fitness model for the Italian interbank money market. Phys Rev E 74:066112

50. Boguñá M, Pastor-Satorras R (2003) Class of correlated random networks with hidden variables. Phys Rev E 68:036112

51. Gautreau A, Barrat A, Barthélemy M (2009) Microdynamics in stationary complex networks. Proc Natl Acad Sci USA 106:8847-8852

52. Cocco JF, Gomes FJ, Martins NC (2009) Lending relationships in the interbank market. J Financ Intermed 18:24-48

53. Hatzopoulos V, lori G, Mantegna RN, Miccichè S, Tumminello M (2015) Quantifying preferential trading in the e-mid interbank market. Quant Finance 15:693-710

54. Temizsoy A, lori G, Montes-Rojas G (2015) The role of bank relationships in the interbank market. J Econ Dyn Control 59:118-141

55. Kobayashi T, Takaguchi T (2017) Identifying relationship lending in the interbank market: A network approach. arXiv:1708.08594

56. Squartini T, Garlaschelli D (2011) Analytical maximum-likelihood method to detect patterns in real networks. New J Phys 13:083001

57. Squartini T, van Lelyveld IV, Garlaschelli D (2013) Early-warning signals of topological collapse in interbank networks, Sci Rep 3:3357

58. Mastrandrea R, Squartini T, Fagiolo G, Garlaschelli D (2014) Enhanced reconstruction of weighted networks from strengths and degrees. New J Phys 16:043022

59. Squartini T, Mastrandrea R, Garlaschelli D (2015) Unbiased sampling of network ensembles. New J Phys 17:023052

60. Cimini G, Squartini T, Gabrielli A, Garlaschelli D (2015) Estimating topological properties of weighted networks from limited information. Phys Rev E 92:040802

61. Cimini G, Squartini T, Garlaschelli D, Gabrielli A (2015) Systemic risk analysis on reconstructed economic and financial networks. Sci Rep 5:15758

62. Anand K et al (2017) The missing links: a global study on uncovering financial network structures from partial data. J Financ Stab 35:107-119

63. Godoy-Lorite A, Guimera R, Sales-Pardo M (2016) Long-term evolution of email networks: statistical regularities, predictability and stability of social behaviors. PLoS ONE 11:e0146113

64. Bajardi P, Barrat A, Natale F, Savini L, Colizza V (2011) Dynamical patterns of cattle trade movements. PLoS ONE 6:e19869

65. https://graph-tool.skewed.de/

66. Estrada E, Rodríguez-Velázquez JA (2005) Spectral measures of bipartivity in complex networks. Phys Rev E 72:046105

\section{Submit your manuscript to a SpringerOpen ${ }^{\circ}$ journal and benefit from:}

- Convenient online submission

- Rigorous peer review

- Open access: articles freely available online

- High visibility within the field

- Retaining the copyright to your article

Submit your next manuscript at $>$ springeropen.com 